\section{Neupane $Y^{1}$}

Paudel D R 2

Ganesh Man Singh Memorial Academy of ENT and Head \& Neck Studies, Tribhuvan University Teaching Hospital, Institute of Medicine, Kathmandu, Nepal. ${ }^{1}$

Department of ENT-HNS

Bharatpur Hospital, Chitwan, Nepal. ${ }^{2}$

Correspondence to:

Dr .Dhundi Raj Paudel

Department of ENT-HNS

Bharatpur Hospital, Chitwan, Nepal

E-mail: dhundi_paudel@yahoo.com

\section{CARCINOMA IN A THYROGLOSSAL DUCT CYST}

Abstracts:

Thyroglossal duct cyst (TGDC) is most common congenital benign cervical mass. Carcinoma arising in the TGDC accounts for only about 0.7 to $1.5 \%$ of all TGDC. Presentation of TGDC carcinoma is similar to that of benign duct cyst. Papillary carcinoma is common carcinoma in TGDC, median age of presentation is 40 years, with female to male ratio of 3:2. Diagnosis of TGDC carcinoma is often based on pathological analysis of masses. FNAB have only $53 \%$ true positive rate in a diagnosis. USG, CT scan, thyroid scan play important role in diagnosis of malignancy. Sistrunks' procedure, total thyroidectomy with or without lymphnode dissection and treatment with radioactive iodine have to considered according to clinical and microscopic finding.

Key words: Thyoglossal duct cyst, Papillary carcinoma, Sistrunks' procedure

\section{INTRODUCTION:}

Thyroid gland develops from a median thickening in the floor of the embryonic pharynx just caudal to tubercular impar. The thickening forms the thyroid diverticulum, which descends to the front of the neck, but a connection to foramen caecum of the tongue persists as the thyroglossal duct. By the eight week of embryonic life, bilobed thyroid attains its final position in front of trachea and upper end of duct usually disappears, but the tissue around the lower end sometimes persists as the pyramidal lobe of thyroid gland. Sometime duct may fail to disappear and persists as thyroglossal duct cysts anywhere from foramen caecum to thyroid gland. During the descent of thyroid gland and thyroglossal duct, hyoid bone comes into close relationship with thyroglossal duct. So the hyoid bone might completely surround the duct or might displace the duct, positioning the duct either anterior or posterior to hyoid bone or the duct might end at the hyoid bone. Therefore if excision of thyroglossal duct is necessary, the tract of duct must be dissected free of hyoid bone, or a block of central bone must be taken to ensure that duct is completelyremoved. Thyroglossal duct cyst are most frequent congenital cervical anomaly that occurs in $7 \%$ of adult population. ${ }^{1}$ Carcinoma arising in the thyroglossal duct cyst accounts only for about 0.7 to $1.5 \%$ of all thyroglossal duct cyst. Synchronous occurrence of thyroglossal duct cyst carcinoma and thyroid carcinoma are reported even rarer. Presentation of thyroglossal duct cyst carcinoma is similar to that of a benign duct cyst, that may manifest as a midline neck mass. In general diagnosis of TGDC carcinoma is based on the pathological examination of mass, but needle aspiration cytology, ultrasound and CT Scan play a role in the differential diagnosis, staging and extension of carcinoma and then in the planning of primary treatment. As TGDC carcinoma is rare, there is a great deal of controversy regarding the treatment. Surgical approach is based on individual experience.

\section{DISCUSSION:}

TGDC is the most common benign cervical mass except for benign cervical lymphadenopathy. They present as a cystic midline neck mass that elevates with swallowing and protrusion of tongue. The cyst may be located anywhere along the course of thyroglossal duct cyst. The lesion are usually midline but may be located laterally as well. Thyroglossal duct cyst carcinoma are rare with just more than 250 cases published till date. ${ }^{2}$ Brentano in 1911 and Ucherman in 1915 are credited as being among the first to describe a neoplasm in a thyroglossal duct remaints. Among the malignant thyroglossal duct cyst, $81.7 \%$ are papillary carcinoma, $6.9 \%$ are mixed papillary -follicular carcinoma, $5.2 \%$ are squamous cell carcinoma, $1.7 \%$ are follicular and adenocarcinoma each and $0.9 \%$ are malignant struma, epidermoid carcinoma and anaplastic carcinoma each. ${ }^{3}$ Among the various type of neoplasia in thyroglossal duct cyst, a papillary carcinoma has best favorable prognosis, while a squamous cell carcinoma has the worst prognosis. There are various theories to explain the thyrogenic origin of TGDC carcinomas. Firstly, the de novo theory, that a carcinoma may arise from thyroglossal remnants is based on the fact that in $45 \%$ of cases the presence of thyroid tissue in a thyroglossal duct cyst can be identified histopathologically and this is supported by the absence of a medullary carcinoma in the thyroglossal duct cyst as it arises from parafollicular cell. 4 The second is the metastatic theory which suggests that TGDC carcinoma is a metastatic from an occult primary thyroid gland , as papillary carcinoma is multifocal in nature. ${ }^{5}$ Some author believe that TG duct could act as a natural conduct for the spread of thyroid carcinoma, so, the metastatic theory seems less likely. 6 Other author concede that squamous cell carcinoma is probably the only true carcinoma of TGD, since other malignancies actually develop in ectopic thyroid tissue. ${ }^{7}$

The 3 diagnostic criteria of papillary carcinoma of TGDC are histological identification of TGDC (presence of thyroid follicles in the wall of cyst), the existence of normal thyroid tissue adjacent to tumour and absence of papillary carcinoma in thyroid. 8 Median age of presentation is 40 years of age, although $20 \%$ are less than 20 years of age at the time of presentation. Peak incidence are in 3 rd and $6^{\text {th }}$ decades of life with female to male ratio of 3:2. The presentation is similar to that of benign thyroglossal duct cyst. Cancer of TGDC doesn't have its own symptoms in $70 \%$ of cases. Minority of patients may report pain, tenderness, hoarseness, dysphagia, sinus tract drainage and weight loss. 
Some author suggest a rapid increase in size of TGDC, presence of a firm palpable mass, destruction of hyoid bone, lymphadenopathy, chondrolysis of thyroid cartilage and existence of cold nodule in thyroid gland as a sign of malignancy. Regional lymphnode metastasis of papillary carcinoma of thyroglossal duct cyst occurs much less frequently than in primary cancer of thyroid. Diagnosis of TGDC carcinoma is often based on pathologic analysis of the mass. FNAB has only $53 \%$ true positive rate in a diagnosis. The diagnostic criteria for papillary carcinoma in FNAB are the same whether it arises in TGDC or thyroid proper which include high cellularity, presence of papillary formation and cells with enlarged nuclei with anisonucleosis and powdery chromatin and definitive nucleoli. Intranuclear pseudoinclusions and grooves are significant diagnosis criteria. Psammona bodies, multinucleated grant cells and ropy colloid are variably present. In ultrasonography benign TGDC can be anechoic,homogeniously hypoechoic,homogeniously hyperechoic or heterogenous in appearance,and if calcification is seen within TGDC malignancy needs to be suspected. Systematic USG of neck should also be done for associated thyroid pathology not detected by clinical examination. CT scan is usually carried out in solid, deep-fixed TGDC with associated adenopathy. CT scan of benign TGDC demonstrate unilocular, well-circumscribed, low density mass present anywhere from the base of tongue to superior margin of the thyroid gland. Presence of dense or enhancing mural nodule, irregular calcification or both within the cyst raise the possibility of concurrent malignancy. MRI characteristics vary depending on the protein contents of fluid within cyst.

There is a great deal of controversy regarding the treatment of TGDC carcinoma as it remains unclear whether additional surgery such as thyroidectomy and lymphnode dissection is necessary or not. Full knowledge of the embryogenesis of the thyroid gland and pathology of carcinomas arising in the structures is mandatory for the appropriate management of thyroglossal duct diseases. Few authors consider the thyroglossal duct as a natural way of spread of an occult thyroid cancer and also recommend total thyroidectomy with neck dissection but others believe that the malignancy arises directly in the thyroglossal duct cyst and consider surgical excision of the cyst as curative, at least in some cases. ${ }^{10}$ The distinction between primary carcinoma arising from ectopic thyroid tissue and metastatic disease from an occult or manifest thyroid gland carcinoma is very important in therapeutic decision making but it is often difficult to achieve, therefore significant attention must be given to preoperative evaluation which should include a complete physical examination, thyroid function test, thyroid scan, USG, CT scan and FNAB.

Some author advocate sistrunks' procedure alone as first surgery of choice which include enbloc resection of TGDC with hyoid bone and surrounding soft tissue towards the foramen caecum in patients younger than 45 years with clinically or radiologically normal thyroid gland (i.e. microscopic carcinomatous foci without invasion of the wall of cyst) , small tumour with an absence of histologically positive margins, absence of palpable regional lymph node metastasis and no history of neck irradiation in childhood. ${ }^{9}$ Patel et al, have found that patient with sistrunks' procedure have significantly better 10 years survival compared to patient who had simple thyroglossal cyst excision (100\% versus $75 \%$ respectively) for TGDC carcinoma. ${ }^{11}$ Although sistrunks' procedure is a standard procedure for TGDC carcinoma, the actual extend of surgery depend on the degree of tumour involvement as detected during surgery or thyroid scan. If thyroid gland and lymphnode are grossly normal during sistrunks' procedure, a post operative thyroid scan is recommended. If however a thyroid scan is abnormal or if either thyroid nodules or a mass is identified during surgery, total thyroidectomy with central compartment dissection is recommended.
In a series by Dzodic et al, $25 \%$ of patient had synchronous thyroid gland lesion out of 12 TGDC carcinoma patient. Another series by Hartl et al, reported synchronous lesion in thyroid in 9 out of 16 TGDC carcinoma patients (56\%), who underwent sistrunks' procedure and thyroidectomy, bilateral lobes were involved in 3 patients. 12 So total thyroidectomy could be a good alternative for TGDC carcinoma patients to decrease the recurrence rate and to make post operative followup easier by monitoring thyroglobulin level. ${ }^{13}$ Surgical strategy regarding lymphnode is also controversial . Most TGDC carcinoma is papillary type which frequently metastasize to regional lymphnode. Ultrasonography is the most useful tool to preoperatively detect and diagnose lymphnode metastasis. But the lymphnode metastasis both in level II- IV and VI were frequently overlooked on preoperative USG in study done by Ito et al. ${ }^{14}$ level I is the nearest compartment from TGDC. In the study by Dzodic et al, 5 of 11 patient who underwent prophylactic level I dissection for TGDC carcinoma showed postoperative lymphnode positive, so level I dissection is very important for TGDC carcinoma surgery. Whether and how level II IV dissection should be performed or not depends on the results of frozen section of level II/III. ${ }^{15}$ Some surgeons perform Modified radical neck dissection for patient positive at level II and III during frozen section but not routinely as reoperation for the recurrence to lateral neck region is not very difficult, but reoperation for level VI recurrence is problematic, so central neck dissection is advised prophylatically in patient with nodules suspected of synchronous carcinoma in the thyroid gland. Latent metastasis might not be immediately life threatening, but it has been shown that in papillary carcinoma larger than $1 \mathrm{~cm}$, metastasis to level $\mathrm{Vl}$, which is nearest from the thyroid, independently predicted a worse disease free survival. ${ }^{16}$ Postoperative radioactive iodine ablative therapy should be given to patients with TGDC carcinoma and thyroid carcinoma. In case of local recurrence or metastasis disease, further surgery, postoperative external beam radiation or radioactive iodine therapy should be employed as need. As thyroid tissue in a cyst is also sensitive to TSH, majority of author recommend thyroid suppression with L-thyroxin for all patients with papillary TGDC carcinoma independently of the presence of normal thyroid scan or whether patient undergo thyroidectomy to prevent the thyroid tissue stimulation, tumoral or normal. Prognosis of papillary carcinoma is identical to that of papillary carcinoma of thyroid, with rate of curability of $95 \% .{ }^{17}$ In cases where the thyroidectomy is not carried out, long term monitoring is necessary to detect a latent thyroid cancer.

\section{CONCLUSION:}

Thyroglossal duct cyst is most common neck swelling usually located in the midline of neck. The coexistence of carcinomas in TGDC is extremely rare so the diagnosis of TGDC carcinoma can be missed due to its rarity. The cyst is usually asymptomatic and the presentation of the patient with carcinoma is indistinguishable from benign TGDC. Diagnosis is only made postoperatively after excision of the cyst. TGDC carcinoma generally shows a favorable prognosis. Sistrunks' procedure, total thyroidectomy with or without lymphnode dissection and treatment with radioactive iodine have to be considered according to clinical and microscopic finding. so accurate preoperative clinical and radiological evaluation be performed in order to plan surgical strategy.

\section{REFERENCES:}

1. Ellis et al, The applied anatomy of thyroglossal tract remants, larynscope, 1997,87: 765-770

2. Dan et al, A case of malignancy in thyroglossal duct cyst-recommendation for management. J Natl Med Assoc.2012 Mar-Apr : 104(3-4): 211-4 
3. Weiss SD, orlich CC. Papillary carcinoma of a thyroglossal duct cyst; report of a case and literature review; Br J surg. 1991: 78: 87-89

4. Sorrenti G, Cavazzuti PP, Zanetti G: papillary carcinoma arising in thyroglossal duct cyst; a case report and review of the literature. Acta Otorhinolaryngol Ital 1995,15: 460-464

5. Tew S, reeve TS, Poole AG, Delbridge L. Papillary thyroid carcinoma arising in thyroglossal duct cyst: incidence and management. Aust NZ J Surg.1995; 65: 717-718

6. Crile G, Jr Papillary Carcinoma of the thyroid and lateral cervical region;so called lateral aberrant thyroid. Surg Gynecol Obstet. 1994; 85:757-766

7. Boswell WC, Zoller M, Williams JS,Lord SA, Check W. thyroglossal cyst carcinoma. J Laryngol Otol.1984;98:1277-1280.

8. Wind Strom et al, Adenocarcinoma originating in the thyroglossal duct, Ann Otol Rhinolaryngol,1976, 85:286-290

9. Mazzarefi El (2004). Thyroid cancer In TG Duct remants: a diagnostic and therapeutic delimma. Thyroid 14:335-336

10. Ash et al. Coincidence of thyroid tumour and thyroglossal duct remants. Review of literature and presentation of 3 cases. Tumori.2003; 89;312-32.

11. Patel et al (2002). Management of well differentiated thyroid carcinoma presenting within a thyroglossal cyst. J Surg Oncol 79: 134-139
12. Hartl et al.(2009) High risk of multifocality and occult lymphnode metastases in papillary thyroid carcinoma arising in thyroglossal duct carcinoma; reflection of a series of eighteen cases. Thyroid 14: 121:123.

13. Miccoli et al(2004) extent of surgery in thyroglossal duct carcinoma: reflection on a series of 18 cases. Thyroid 14; 121-123

14. Ito et al (2007). Risk factor for recurrence to the lymph node in papillary thyroid carcinoma patients without preoperatively detectable lateral node metastasis: validity of prophylactic modified radical neck dissection. World J surg 31(11): 2085-2091

15. Ito et al. (2006) Clinical significance of lymphnode metastasis of thyroid papillary carcinoma located in one lobe. World J Surg 30:1821-1828.

16. Kermani et al, Papillary carcinoma arising in a thyroglossal duct cyst: case report and discussion of management modalities, Eur Arch Otorhinolaryngol,2000, 265:233-236

17. Dzodic et al (2006). Sentinel lymphnode biopsy may be used to suppert the decision to perfrom modified radical neck dissection in differentiated thyroid carcinoma. World J surg 30(5);841-846. 